\title{
Uncommon skeletal metastasis secondary to transitional cell carcinoma
}

\author{
Andrea Melilli* \\ 224 Hardcastle Place, Valrico, FL 33595, USA
}

\begin{abstract}
Background: Canine transitional cell carcinoma (TCC) is the most common type of malignant tumor of the urinary system. This tumor rarely metastasizes to bones, and dogs most commonly present with lower urinary tract signs.

Case Description: An 8-year-old female spayed beagle presented with unilateral front-limb lameness in which pain was localized to the shoulder joint. Surgical bone biopsy following abnormal radiographic lesions noted in the scapula was consistent with metastatic carcinoma of unknown origin. Abdominal ultrasound revealed a mass in the trigone region of the urinary bladder, which was cytologically confirmed to be a transitional cell carcinoma. Amputation was elected due to severity of the limb pain, and chemotherapy for the TCC was scheduled to follow after healing from surgery was complete. At the 2-week recheck for commencement of chemotherapy, the patient was anemic and a pathologic fracture was found on the L5 vertebra. Due to poor prognosis, the owner elected for humane euthanasia.

Conclusion: This case report documents the clinical and pathological findings of a dog where metastasis to a rarely documented distant bone site secondary to TCC was diagnosed before the primary tumor. The unusual sequence of events described in this case report is a reminder to always consider all the possible differential diagnoses, as that may influence our recommendation on diagnostics and treatment.
\end{abstract}

Keywords: Bone metastasis, Dog, Transitional cell carcinoma.

\section{Introduction}

Transitional cell carcinoma (TCC) is the most common malignant tumor of the urinary tract in dogs (Mutsaers et al., 2003). Most dogs present with lower urinary tract signs, such as, but not limited to, stranguria, pollakiuria, dysuria, and hematuria. In cases with lower urinary tract signs, diagnosis of TCC may be expedited by the factors like availability of ultrasound in general practice, no evidence of concurrent urinary tract infection (Allstadt et al., 2014) and breed predilection (Norris et al., 1992). Lack of urinary tract signs is rare, causing a significant delay in the final diagnosis and treatment of TCC. Metastasis of TCC to the bone is uncommon; with most common documented sites involving the femur and vertebrae although metastasis to ribs, skull, humerus, and radius have been documented (Ruby et al., 1989; Johnson et al., 2003; Malek et al., 2011; Colledge et al., 2013). This report presents a unique TCC case of a dog lacking lower urinary tract signs, and where bone metastasis to a rarely documented site was diagnosed prior to the primary tumor.

\section{Case Details}

An 8-year old female spayed beagle presented with a complaint of intermittent left front limb lameness of 4-day duration. Obvious lameness was not appreciated on physical examination; however, considerable pain was apparent upon palpation of the humeroscapular joint. The remainder of the physical examination was normal with the exception of dental calculus and a body condition score of 7/9. Radiographic study under sedation was recommended, but the client opted for symptomatic treatment of pain and bed rest. Tramadol was prescribed at $4.6 \mathrm{mg} / \mathrm{kg}$ every 12 hours for 14 days. The patient returned 8 days later for persistent lameness. Per the client, Tramadol was given at the recommended dosage for three consecutive days, but was discontinued due to lack of improvement. Pain was again elicited on palpation of the left humeroscapular joint, as well as with its extension and flexion. Radiographs with consult were approved at this visit, which revealed multiple small regions of radiolucency in the distal aspect of the spine of the left scapula, including the acromion process (Fig. 1). Radiographic consult by a board-certified radiologist stated a concern for an aggressive lytic lesion; possibly representing a neoplastic process such as a primary mesenchymal tumor or a round cell tumor. The radiolucencies were subtle, so the possibility that it represented a superimposition artifact could not be ruled out. The radiologist recommended an ultrasound evaluation of the affected scapula. If a cortical irregularity were to be identified on ultrasound, bones aspirates should be obtained for cytologic evaluation. Alternatively, follow-up radiographs were suggested to be evaluated in 3-5 week to reassess the appearance of the left scapula. Carprofen was prescribed at $2.2 \mathrm{mg} / \mathrm{kg}$ twice daily for 7 days to evaluate the patient's response while awaiting the client's decision. The client called 2 weeks after the last appointment stating she had taken the patient to a different veterinary clinic where a second set of radiographs were taken, which revealed a more pronounced radiolucency in the left scapula (images not available due to analog radiographs being taken at a different clinic). 


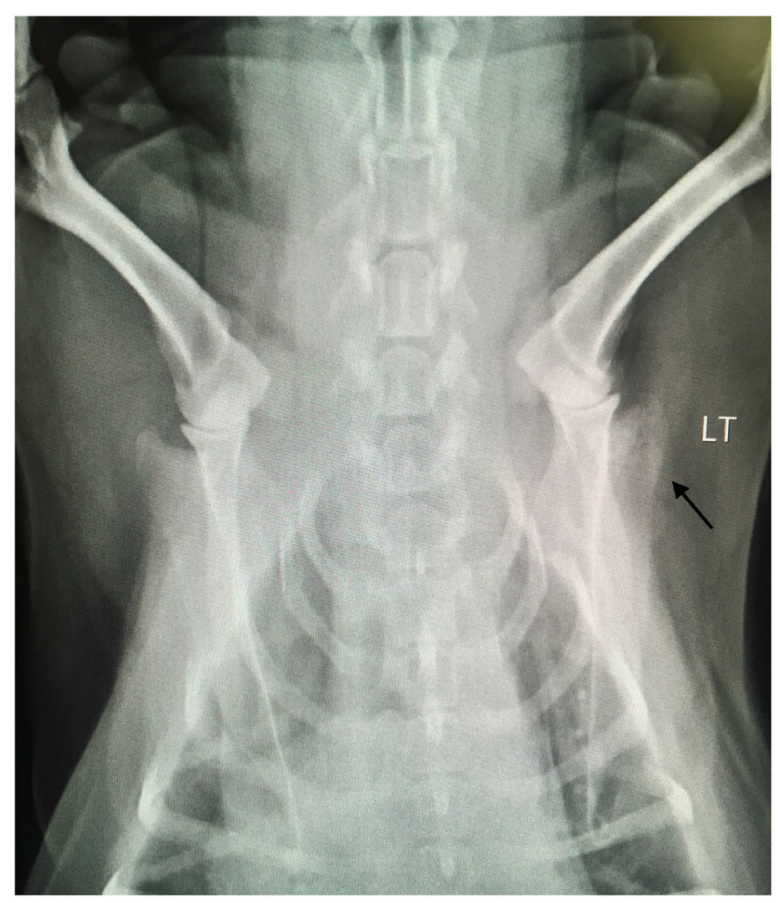

Fig. 1. Radiograph showing multiple small regions of radiolucency in the distal aspect of the spine of the left scapula, including the acromion process (arrow). LT $=$ left.

Due to the severity of the progressing limb pain, the client opted to perform bone biopsy under general anesthesia versus ultrasound-guided cytology, as the cost of the latter at a specialty clinic was considerably more than the former at the general practice. A fentanyl patch of $50 \mathrm{mcg} / \mathrm{hour}$ was prescribed and applied prior to the bone biopsy procedure performed the following day. Blood work performed prior to general anesthesia showed a slight increase in hematocrit of $55.21 \%$ [reference range (RR) $37.0 \%-55.0 \%$ ] and chemistry showed a mild to moderate increase in the levels of alkaline phosphatase $405 \mathrm{U} / \mathrm{l}$ (RR 23.0-212.0 U/l) and a slight decrease in the levels of globulin $2.2 \mathrm{~g} /$ dl (RR 2.5-4.5 g/dl). Electrolytes levels were within the reference range. A bone biopsy was performed with J-type trephine to acquire three samples from the proximal scapula, which were submitted for histopathology. Anesthesia and recovery for the procedure were uneventful. Gabapentin at $7 \mathrm{mg} / \mathrm{kg}$ twice daily and Carprofen at $2.2 \mathrm{mg} / \mathrm{kg}$ twice daily were prescribed in addition to the Fentanyl patch (changed every 72 hours) for supplemental pain relief. The owner reported that the patient's pain was well controlled with the combination of all three drugs.

Laboratory results returned a few days later, and the microscopic description of the bone biopsy was reported as follows: infiltrating, what appears to be reactive woven bone and skeletal muscle, is a mass composed of polygonal cells arranged in cords and clusters. Cells have round nuclei, finely stippled chromatin, inconspicuous nucleoli, a moderate amount of amphophilic cytoplasm, and distinct cell borders. Anisocytosis and anisokaryosis are mild to moderate. Mitoses are occasionally observed. Cells appear to occasionally surround a lumen filled with lightly basophilic material. Osteoclasts are occasionally observed within Howship's lacunae. The biopsy results were reviewed by three board-certified pathologists and concluded to be a metastatic carcinoma. Primary carcinoma was suggested to be originating from mammary gland, urinary bladder, or prostate tissue.

Ten days following the bone biopsy procedure, the client consulted with a board-certified oncologist. The patient had lost $1.76 \mathrm{~kg}$ from the time of initial presentation (approximately 5 weeks), but besides left front limb lameness, no other significant abnormalities were noted on physical examination. An abdominal ultrasound was performed on the same day by the oncology specialist, which revealed a mass in the trigone region of the urinary bladder. The remainder of the abdominal ultrasound was normal. The bladder mass was aspirated percutaneously and sent out for the cytologic evaluation. With cytology results pending, the subsequent options for pain control and improved quality of life were offered: 1) left front limb amputation, 2) radiation, or 3) bisphosphonate therapy plus continued oral pain medication in combination with a fentanyl patch. Chemotherapy was also discussed, pending cytology results. Due to the severity of the front limb pain, the owner opted for limb amputation. Carprofen was replaced with Piroxicam at $0.26 \mathrm{mg} / \mathrm{kg} /$ day, and Gabapentin and Fentanyl patch continued as previously prescribed.

Amputation was scheduled to be performed the following day as long as there was no radiographic evidence of metastasis to the unaffected limbs. Radiographs of the hind limbs were taken and included lumbar vertebrae 6 and 7, pelvis, femur, tibia, and proximal metatarsal bones. Non-affected right front limb radiographs included cervical vertebrae $2-6$, thoracic vertebrae 1-11, scapula, humerus, radius, and ulna. All included bones were reviewed and deemed free of obvious metastatic disease on radiographs. Blood work prior to general anesthesia for limb amputation was again performed and revealed similar abnormalities as the previous blood work. Immediately prior to initiating surgery, the oncologist called with the cytology report of the bladder mass. The microscopic findings of the needle aspirate of the urinary bladder were reported as follows: one smear is large and densely cellular with abnormal epithelial cells, which are spread in a monolayer. The epithelial cells are rounded and slightly polygonal and have round nuclei, fine chromatin, multiple nucleoli, blue cytoplasm, and a higher nuclear to cytoplasm ratio than normal epithelial cells. There is moderate variation in sizes of cells, nuclei, and nucleoli, including some large cells with large single nucleus and cells with more than on nucleus. Mitotic cells are easily found. A few cells contain round, granular, pink 
inclusions. One small smear also contains abnormal cells. Additional comments communicated that the location of the mass and morphology of the neoplastic cells were consistent with transitional cell carcinoma. The client was informed of the results by the oncology specialist and opted to proceed with the planned front left limb amputation. Chemotherapy was scheduled with the oncology specialist 2 weeks post-surgery. No complications occurred during surgery or recovery. The patient was discharged on the same day with a new Fentanyl patch (q72h) in place. The same dosing for Gabapentin and Piroxicam were continued.

Fourteen days after surgery, the patient presented to the oncology specialist for suture removal and initiation of Mitoxantrone for TCC. The owner reported that the patient had been progressively weaker since the limb amputation and had been experiencing episodes of pale mucous membranes. On physical examination, the patient was down, mucous membranes were pale, and had a painful abdomen. A work-up consisting of a complete blood cell count (CBC), abdominal ultrasound and abdominal radiographs were performed prior to chemotherapy administration. CBC showed a hematocrit of $14 \%$ (RR 37.0\%-55.0\%), white cell count of $29,000 \mu \mathrm{l}(\mathrm{RR} 6,000-17,000 \mu \mathrm{l})$, and neutrophilia at $13,000 \mu \mathrm{l}(\mathrm{RR} 2,000-10,600 \mu \mathrm{l})$. The severe anemia was suspected to be due to a gastrointestinal ulcer induced by Piroxicam administration. The need for a blood transfusion was discussed, as well as a repeat of an abdominal ultrasound and radiographs, if moving forward with chemotherapy.

Abdominal ultrasound revealed extreme thickening of the stomach, no overt free fluid, and enlargement of the urinary bladder mass. Abdominal radiographs revealed no free gas presents; however, a lytic lesion on the L5 vertebra with a secondary pathologic fracture was detected. Although the affected vertebra was not biopsied, it was suspected to be another distant metastatic site from the TCC.

After discussing the aggressive progression of metastatic disease resulting in a pathologic fracture of the L5 vertebrae, giving the patient an extremely poor quality of life, the owner opted for humane euthanasia.

\section{Discussion}

Many different types of treatments have been used to clinically control transitional cell carcinomas although all have been palliative thus far (Simmons et al., 2015). Treatment options include surgery, laser, radiation, chemotherapy, non-steroidal anti-inflammatory drugs, biphosphate therapy, or a combination of these (Mutsaers et al., 2003). Choice of treatment is influenced by factors, such as location/size of the mass, clinical signs, finances, and availability of diagnostic tools, specialists, and drugs.

This case posed many difficulties in diagnosis and treatment choice. First, it did not present with any of the most common clinical signs of TCC (urinary signs).
Second, the severity of limb pain leads us to address the metastatic disease first, which delayed administration of chemotherapy to target the primary TCC tumor. In addition, the adverse gastrointestinal effects (suspected to be from Piroxicam administration) leading to significant anemia, as well as a second metastatic site resulting in a pathologic fracture, completely altered the planned course of treatment.

This case report highlights the importance of performing thorough preliminary diagnostics. Despite the presence or lack of specific clinical signs, basic diagnostics should always include blood work (CBC/serum or plasma biochemical analysis/electrolytes), radiographs (chest and abdomen), and urinalysis (+/- urine culture). Perhaps neoplastic cells would have been detected in the urine sediment if collected via catheterization (Norris et al., 1992), which would have led us to perform an abdominal ultrasound prior to considering an invasive bone biopsy. This scenario would likely be more promising in a male dog, as urinary catheterization in females can be extremely difficult, especially without sedation or general anesthesia. Due to this known challenge, it is suspected that the individual sampling the bladder mass chose to perform percutaneous sampling instead. Although the patient did not live long enough to encounter any issues related to the chosen method of sampling, we must always take into consideration the possibility for diffusion of neoplastic cells throughout other tissues when performing a percutaneous aspirate of a malignant mass. In the efforts to avoid potential tumor seeding, urinary catheterization under sedation/ anesthesia should have been considered as a superior alternative in collecting a sample of the bladder mass in this case (Fulkerson and Knapp, 2015).

In retrospect, the entire vertebral column should have been included in the radiographs prior to limb amputation although lesion of L5 may not have been grossly visible at that time. Since TCC metastasis has now been documented in numerous different tissues in the body (Ruby et al., 1989; Mutasers et al., 2003; Malek et al., 2011; Colledge et al., 2013; Reed et al., 2013; Simmons et al., 2015; Charney et al., 2017), computed tomography should probably be considered in cases with distant metastasis prior to radical treatment; albeit the cost and availability may be of hindrance. In the above case, computed tomography could have detected subtle changes in bones (and possibly other tissues), therefore changing the course of treatment entirely.

\section{References}

Allstadt, S.D., Lee, N.D., Scruggs, J.L., Bernard, J., Hecht, S., Callens, A., Seibert, R. and Bartges, J.W. 2014. Clinical rounds: transitional cell carcinoma. Available via http://www.veterinarymedicine. dvm360.com/clinical-rounds-transitional-cellcarcinoma (Accessed 22 August 2018).

Charney, V.A., Miller, M.A., Heng, H.G., Weng, H.Y. and Knapp, D.W. 2017. Skeletal metastasis 
of canine urothelial carcinoma: pathologic and computed tomographic features. Vet. Pathol.54, 380-386.

Colledge, S.L., Raskin, R.E., Messick, J.B., Tiffany, R.L., Wigle, W.L. and Balog, K.A. 2013. Multiple joint metastasis of transitional cell carcinoma in a dog. Vet. Clin. Pathol. 42, 216-220.

Fulkerson, C.M. and Knapp, D.W. 2015. Management of transitional cell carcinoma of the urinary bladder in dogs: a review. Vet. J. 205(2), 217-225.

Johnson, K.D., Character, B.J. and Tyler, J.W. 2003. What is your diagnosis? Trabecular bone destruction in the distal humeral metaphysis and the proximal radial metaphysis. J. Am. Vet. Med. Assoc. 222, 937-938.

Malek, S., Murphy, K.A., Nykamp, S.G. and Allavena, R. 2011. Metastatic transitional cell carcinoma in proximal humerus of a dog. Can. Vet. J. 52, 10131017.
Mutsaers, A.J., Widmer, W.R. and Knapp, D.W. 2003. Canine transitional cell carcinoma. J. Vet. Intern. Med. 17(3), 136-144.

Norris, A.M., Laing, E.J., Valli, V.E., Withrow, S.J., Macy, D.W., Ogilvie, G.K., Tomlinson, J., McCaw, D., Pidgeon, G. and Jacobs, R.M. 1992. Canine bladder and urethral tumors: a retrospective study of 115 cases (1980-1985). J. Vet. Intern. Med. 6, 145-53.

Reed, L.T., Knapp, D.W. and Miller, M.A. 2013. Cutaneous metastasis of transitional cell carcinoma in 12 dogs. Vet. Pathol. 50, 676-681.

Ruby, E.P., Richard, E.W., Gerald, E.D. and James, F.P. 1989. Transitional cell carcinoma of the bladder with skeletal metastases in a dog. J. Am. Anim. Hosp. Assoc. 25(5), 547-551.

Simmons, J.K., Hildreth III, B.E., Supsavhad, W., Elshafae, S.M., Hassan, B.B., Dirksen, W.P., Toribio, R.E. and Rosol, T.J. 2015. Animal models of bone metastasis. Vet. Pathol. 52, 827--841. 